\title{
Cross-talk of different dimensional methylation modification patterns alters tumor microenvironment infiltration and immunotherapy response in renal cancer
}

\section{Xin Qin}

Shandong University Qilu Hospital https://orcid.org/0000-0003-4931-9752

\section{Ling Pan}

Shandong University Qilu Hospital

\section{Lei Yan}

Shandong University Qilu Hospital

\section{Zhiqing Fang}

Shandong University Qilu Hospital

Yidong Fan ( $\square$ fanyd@sdu.edu.cn )

Shandong University Qilu Hospital

\section{Research}

Keywords: Cross-talk, Methylation modification, Tumor microenvironment, Mutation burden, Immunotherapy

Posted Date: September 28th, 2021

DOl: https://doi.org/10.21203/rs.3.rs-921670/v1

License: (c) (i) This work is licensed under a Creative Commons Attribution 4.0 International License. Read Full License 


\section{Abstract \\ Background}

Different dimensional methylation modifications, including DNA, RNA and histone methylation, are mediated by different functional regulators and play an indispensable role in tumor progression. However, the cross-talk and underlying mechanisms of these modification patterns in tumorigenesis, tumor microenvironment (TME) disorder and aberrant immune response are still unknown.

\section{Methods}

We summarized the 9 most common types of modifications from different dimensions and revealed transcriptional and genetic alterations of 104 modification regulators in clean cell renal cell carcinoma (ccRCC) patients. Next, we constructed a prognosis-correlation network to further explore their interactions and identified distinct methylation modification patterns by using unsupervised clustering algorithm. Lastly, we constructed a methylation modification regulator score (MMR Score) model to quantify the characterizations of different modification patterns for individual patients, and validated its predictive value to immunotherapy response.

\section{Results}

We investigated the interactions between different dimensional modifications and identified critical negative regulated nodes in the prognosis-correlation network. Our clustering results revealed that, different from other cancers, immune activity in ccRCC not only depended on the abundance of immune cells, but also the infiltration of stromal cells. The MMR Score for the quantification of each patient can act as an effective biomarker to predict clinical outcomes. Patients with high MMR Score were more likely to experience a therapeutic advantage and clinical benefits from immunotherapy.

\section{Conclusions}

This work revealed that cross-talk of different modifications could lead to the complexity and heterogeneity of individual TME. The integrated quantification of individual modification patterns can better guide clinical personalized cancer treatments.

\section{Background}

The global incidence and mortality of renal cancer have been steadily increasing in the past few years [1]. Clean cell renal cell carcinoma (ccRCC) is the most common pathological type of adult renal cancer and rarely presents symptoms in the early stages of tumorigenesis [2]. Surgical resection is the primary option for clinical treatment, but $30 \%$ of patients will still experience postoperative recurrence and distant 
metastasis, which make five-year survival rates decline from 69.4-10\% [3]. Although the successful application of immunotherapy and targeted therapy brings many new opportunities, the high heterogeneity of ccRCC and limited application scope remain an unsolved problem [4]. Therefore, clarifying the complicated mechanisms of drug resistance and finding new biomarkers to guide clinical therapy is urgently needed.

Epigenetics is an important component of genetics that leads to stable and heritable phenotypes but does not interfere with nucleotide sequences and Watson-Crick pairing [5]. It greatly enriches genetic central dogma and makes gene regulation more complicated [6]. The process is dynamic and reversible and regulated by a complex network of modification regulators [7] Methylation modification is the most common form of epigenetics and mainly focuses on three dimensions: DNA modification, RNA modification and histone modification [6]. One of the most predominant types of DNA modification is 5methylcytosine $(5 \mathrm{mc})$ : methylated at the 5 th carbon of cytosine [8]. The establishment and maintenance of $5 \mathrm{mc}$ play critical roles in life processes. It can regulate gene transcription and chromatin structure and maintain genome stability [9]. Recently, a growing number of studies have reported that RNA modification, especially $\mathrm{N}^{6}$-methyladenosine (m6A), contributes to transcript stability, pre-mRNA splicing, mRNA export and translation initiation [10]. Methylation on chromosome-associated regulatory RNAs (carRNAs), including enhancer RNAs and promoter-associated and repeat RNAs, is also a new breakthrough to prove that RNA modification can regulate chromatin state and gene transcription [11]. Histone methylation is a universal post-translational modification. It is implicated in pre-mRNA splicing, DNA damage signaling, mRNA translation and especially can affect chromatin accessibility [12]. The regulation of epigenetics depends on the coordination between methyltransferases (writers), demethylases (erasers) and binding proteins (readers). The complicated regulatory networks of methylation modifications provide a lot of opportunities for their cross-talk. Meanwhile, cross-talk occurs not only within the same dimension but also between different external dimensions [13]. For example, H3K36me3, directly recognized and bound by METTL14, could guide m6A modification to increase cell stemness globally [14]. Moreover, m6A reader YTHDC1 can interact with KDM3B and recruits KDM3B to m6A-associated chromatin regions to promote H3K9me2 demethylation and gene expression [15]. Furthermore, the ring E3 ubiquitin ligase UHRF1 controls DNA methylation through maintaining DNA methyltransferase DNMT1 to newly replicated chromatin [16]. Therefore, to fully understand the significance of epigenetic regulatory networks, the exploration of cross-talk between different dimensional methylation modifications is urgently needed.

Immunotherapy, especially immune checkpoint blockade (ICB), has been proved to be an effective treatment in many cancers. Although immunotherapeutic drugs such as anti-PD-1 and anti-PD-L1 has shown outstanding clinical efficacy, only a few people exhibit durable responses, which is far short of clinical demands [17]. Consequently, new therapeutic targets and the mechanism of low response rates demand urgent exploration. The tumor microenvironment (TME) is comprised of tumor cells, immune cells, stromal cells, local cytokines and so on [18]. With the deepening understanding of TME, more evidence has shown that tumor immune infiltration plays a key role in tumor recurrence, metastasis and 
response to immunotherapy [19]. At the same time, numerous studies have reported the close relationship between epigenetic modifications and tumor immune infiltration. YTHDF1 can recognize and bind to methylated sites on the transcripts of lysosomal proteases in dendritic cells and increase its expression. The upregulation of lysosomal proteases can enhance CD8 + T cell infiltration and response to anti-PD-L1 blockade [20]. In addition, specific depletion of METTL3 in dendritic cells can downregulate the expression of CD40, CD80 and IL-12 and weaken the ability to stimulate T cell responses [21]. However, these studies only focused on one or two modification regulators and ignored the cross-talk and interaction of the epigenetic regulatory networks. Hence, comprehensive knowledge of TME immune infiltration mediated by methylation modification regulators would improve our understanding of immune regulation and immunotherapy.

In our research, we summarized 1033 ccRCC samples which came from Gene Expression Omnibus (GEO), Array Express and The Cancer Genome Atlas (TCGA) database. We integrated the genetic and transcriptional profiles of methylation modification regulators in $\mathrm{CCRCC}$ and identified four subtypes of methylation modification regulators. We found that these subtypes were not only related to different abundance of immune cell infiltration, but also presented a remarkable difference of stromal cell infiltration, which suppressed immune cell activation and lead to immune escape. With the differentially expressed genes (DEGs) from differential expression analysis, we established the methylation modification regulator score (MMR Score) model. Using the MMR Score, we quantified the characteristics of different methylation modification patterns for each individual patient and evaluated their response to immunotherapy so as to better guide clinical medication managements.

\section{Methods}

\section{Data source and handling}

The copy number alteration data, somatic mutation data, RNA sequencing data and corresponding clinical information of ccRCC patients were downloaded from TCGA database kidney renal clear cell carcinoma (KIRC) cohort. Other microarray datasets came from GEO database and Array Express database. The detailed data source was described in the section of Availability of data and materials. Totally, 5 datasets including TCGA-KIRC, E-MTAB-1980, GSE46699, GSE53757 and GSE73731 were selected to perform a more in-depth study. Expression data was converted to transcripts per kilobase million (TPM) values [22]. SVA package was used to eliminate batch effects between different datasets [23]. We have further selected 2 immunotherapy datasets. IMvigor210 cohort, an anti-PD-L1cohort, and corresponding clinical information came from http://research-pub.gene.com/IMvigor210Corebiologies. Another immunotherapy cohort dataset was downloaded from a published article (PMID: 32472114) including the datasets of CCRCC patients received anti-PD1 treatment [24].

\section{Unsupervised clustering analysis based on methylation modification regulators}

We screened the expression matrix of 104 methylation modification regulators in the 5 datasets including 1033 ccRCC patients (Table S1). Based on the expression data, ConsensuCluster package was used to 
perform unsupervised clustering analysis [25]. Consensus clustering algorithm was used to guarantee the accuracy of clustering and the operation was repeated 1000 times.

\section{Gene set variation analysis (GSVA)}

The R package "GSVA" was used to perform enrichment analysis to find out the different biological functions [26]. We downloaded gene sets from MSigDB database (http://www.gseamsigdb.org/gsea/index.jsp) including c2.cp.kegg.v7.4.symbols and h.all.v7.4.symbols

\section{TME score and immune infiltration evaluation}

ESTIMATE package was used to calculate stromal score and immune score for each ccRCC patients [27]. We employed two algorithms to separately calculate the abundance of immune cells: single sample gene set enrichment (SSGSEA) and CIBERSORT. In the algorithm of sSGSEA, immune cell gene sets were downloaded from the study of Charoentong. The abundance of immune cells was represented by the enrichment scores. In the algorithm of CIBERSORT, our analysis based on Euclidean and Ward's linkage and repeated 1000 times [28].

\section{Screening of methylation modification related genes}

Limma package was used to screen DEGs between distinct methylation modification regulator clusters [29]. $P<0.001$ was considered as significant DEGs. The intersection of significant DEGs from different clusters was considered as methylation modification related DEGs.

\section{Construction of gene subtypes and score model}

Based on the expression of methylation modification related DEGs, we performed unsupervised clustering and consensus clustering algorithms to construct and validate the methylation modification gene subtypes. Then univariate Cox regression was used to screen prognosis-related genes for further analyses. Following this, principal component analysis was used to reduce the dimension and calculated the prognostic value of different methylation modification related gene signatures. Next, we selected principal component 1 and 2 and used a method analogous to $\mathrm{GGI}$ to identify each patient's score [30]:

Score $=\sum\left(P C 1_{x}+P C 2_{x}\right)$.

$\mathrm{X}$ represents the methylation modification phenotype-related genes.

\section{Statistical analysis}

We used spearman correlation coefficient to evaluate the correlations between methylation modification regulators and tumor-infiltrating immune cells. Survminer package was used to determine the best cutoff of survival analysis (Log-rank test). All statistical analyses were done in GraphPad Prism 7.0 and R 4.0.1 software. $P<0.05$ indicated a statistically significant difference. 


\section{Results}

\section{Landscape of genetic and transcriptional changes of methylation modification regulators in ccRCC}

Drawing from the published studies, the 9 most common types of methylation modification in DNA, RNA and histone dimensions containing a total of 104 regulators were taken into account (Additional 2: Table S1). 20 regulators of $5 \mathrm{mc}$ modification, 23 regulators of m6A modification, 26 regulators of another 6 types of RNA modification and 35 regulators of histone methylation modification were included in the current study $[8,10,13,31,32]$.

To explore the genetic alterations of different methylation modifications, we first focused on the prevalence of somatic mutations in ccRCC patients. The results were shown in Additional 1: Figure S1AD. Among 336 samples in TCGA-KIRC cohort, 7.14\% (24 samples) experienced mutation of DNA methylation regulators. The mutation frequencies of m6A modification regulators and 6 other types of RNA modification regulators accounted for $7.74 \%$ (26 samples) and $4.17 \%$ (14 samples) respectively. $17.86 \%$ (60 samples) experienced mutation of histone methylation modification regulators. Regulators with higher mutation frequencies were summarized in Fig. 1a. The overall somatic mutation frequencies of regulators were lower and only SETD2 (12\%; lysine methylation writer) exhibited a relatively higher mutation frequency. Compared to the wide type group, the expression of SETD2 was downregulated in the mutated group (Fig. 1b). Meanwhile, survival analysis showed that the low expression of SETD2 presented poor overall survival (Fig. 1c).

We further found that copy number variations (CNVs) of these regulators (Additional 1: Figure S1E-H) were prevalent among them, especially in 18 regulators (Fig. 1d). Of these regulators, only YTHDC2, ZBTB38, ADAR and IGF2BP2 showed higher frequencies of CNV gains while others experienced widespread losses of copy number. The positions of copy number alterations on chromosomes of these regulators were shown in Fig. 1e. To inspect whether the copy number alterations had influences on the regulators' mRNA level in ccRCC patients, we then performed differential expression analysis by using pan-cancer data from TCGA database (Additional 1: Figure S2A-D). As shown in Fig. 1f, only 16 regulators were significantly differentially expressed in ccRCC patients $(|\log F C| \geq 0.5 ; P<0.05)$. Of these regulators, 7 were upregulated and 9 were downregulated. Comparing gene expressions with copy number alterations (Fig. 1d and 1g), we found that downregulated genes always showed CNV losses (e.g., KDM1A and SETD2) while upregulated genes experienced CNV gains (e.g., YTHDC2). The results suggested that copy number alterations may play an important role in dysregulation of regulators. At the same time, we noticed that the upregulation of WTAP was accompanied by a high frequency of CNV loss. To explore the reason for these contradictions, we divided the samples into 4 groups according to their CNV values: normal, CNV_Loss, none_ CNV and CNV_Gain. In fact, the CNV loss group had lower expression of WTAP than the normal group (Additional 1: Figure S2E). Nevertheless, contrary to expectations, copy number alterations of IGF2BP2 and TET2 presented the opposite trends with their expression. 
Overall, the biogenesis and development of tumors is an intricate process. CNV and somatic mutations couldn't completely describe the dysregulation of regulators and just offered one possible explanation. Those results showed high heterogeneity, which happened in a landscape of genetic and transcriptional changes of methylation modification regulators in ccRCC. It indicated that the dysregulation of methylation modification regulators had potential values for further study.

\section{Prognostic value and crosstalk patterns of different dimensional methylation modification regulators}

In order to improve the accuracy and specificity of analyses. 1033 ccRCC samples from 5 datasets (TCGA-KIRC, E-MTAB-1980, GSE46699, GSE53757, GSE73731) were selected for a more in-depth study. We divided 104 regulators into 4 categories: DNA methylation modification, m6A modification, other RNA modification and histone methylation modification. Univariate Cox regressions were performed to determine the prognostic value. As shown in Fig. 2a-2b, 60 of 104 modification regulators were significantly associated with prognosis.

As the important part of epigenetics, modifications mediated by these regulators enriched the central dogma. We also wondered if there was any mutual influence between them. We performed correlation analyses to demonstrate the interactions of 104 regulators (Additional 1: Figure S2F-I and S3A-F). In DNA methylation modification $(5 \mathrm{mc})$, all regulators had shown significantly positive correlations (Additional 1: Figure S2F). Unexpectedly, our results showed that high expression of writers were not associated with low expression of erasers, and even exhibited very strong positive correlations. When it comes to RNA modification, in m6A modification, negative correlations had much lower frequencies than positive correlations (Additional 1: Figure S2G). Only m6A reader IGF2BP2 and IGF2BP3 showed negative correlation with many other regulators, particularly m6A erasers FTO and ALKBH5. In other common types of RNA modification, Additional 1: Figure S2H revealed a critical negative regulatory role of NSUN7 (m5C writer). Only NSUN7 presented negative correlations with other regulators including NOP2 (m5C writer), METTL1 (m7G writer) and PUS1 (Pseudouracil writer). We also calculated correlations between other common types of RNA modification and m6A modification (Additional 1: Figure S3D). The results indicated that the positive correlation was still the predominant form, especially between all writers and erasers which was quite beyond our expectations. At the same time, the expression of regulators was not only closely connected in the same category, but also showed a significant correlation in different types of modifications. For example, the m1A modification writer TRMB61B presented very strong correlations with METTL14 (m6A writer) and METTL2A (m3C writer). Even the methylation site or base was completely different, and there was no direct evidence showing that the neighbor sites or the same bases could interfere with each other, but their regulators still presented a high frequency of synergistic effects. In histone modification, PRMT8 (Arginine methylation writer) attracted our attention. It negatively correlated with the vast majority of histone regulators, especially with some Lysine methylation regulators like EHMT2, SETDB1 and KDM4C (Additional 1: Figure S2I). We summarized the results of univariate Cox regressions and correlations and constructed a huge prognosis-correlation network among all DNA, RNA and histone modification regulators (Fig. 2C). $\mathrm{P}<0.0001$ was considered as significant for co-expression relationships. 
Recent studies had reported that crosstalk about different dimensions of methylation modification exerted important biological functions in the occurrence and development of tumors [13]. The existing research was mainly focused on the modifications between DNA methylation and histone methylation. The network suggested that many regulators had shown very strong crosstalk patterns in different dimensions or types of methylation regulators. Positive correlations were dominant and modification writers were not necessarily negatively correlated with modification erasers. Focused on the overall analysis of the network, some regulators (such as PRMT8, IGF2BP2 and especially IGF2BP3) might act as important nodes to negatively regulate the network. The above analyses indicated that the cross-talk among regulators is very important in the process of tumorigenesis and has huge values for further study.

\section{Distinct clusters and immune infiltration characteristics of modification regulators}

We used the R package "ConsensusClusterPlus" to perform unsupervised clustering with the expression data of 104 methylation modification regulators. 1033 ccRCC samples were divided into 4 clusters, which were named as methylation-modification-regulator-cluster A-D (MMRC A-D). Group composition was recorded in Additional 2: Table S5. We drew a heatmap to display the expression distribution of regulators in different subtypes (Fig. 3a). The results showed that most of regulators were significantly upregulated in MMRC A and downregulated in MMRC $C$. Only a few regulators exhibited upregulated expression in MMRC C, such as IGF2BP2, IGF2BP3 and UTY. We performed survival analyses for overall survival and used log-rank test to compare the significance (Fig. 3b). The results showed that the ccRCC patients in MMRC A and D exhibited significant survival advantages than MMRC B and C.

To further investigate the biological functions of different clusters, we performed gene set variation analysis (GSVA) using KEGG gene sets and hallmark gene sets. We drew heatmaps to exhibit the top 20 differences in enrichment between each cluster (Fig3c-3d, Additional 1: Figure S4A-F). MMRC B and C with worse survival outcomes were markedly enriched in stromal activation and tumor proliferation, metastasis pathway including EMT signaling pathway, ECM receptor interaction, inflammatory response, IL6-JAK-STAT3 signaling, KRAS and P53 signaling pathway (Fig. 3c, Additional 1: Figure S4C and S4F). The enriched pathways of MMRC A and D were significantly associated with immune activation and tumor-killing pathways such as interferon alpha response, interferon gamma response and $B$ cell receptor signaling pathway (Fig. 3d, Additional 1: Figure S4D-E).

Many articles have reported that methylation modification was closely associated with immune infiltration $[20,21]$. And based on the results of GSVA, we also found that some immune-related pathways were activated in different clusters. Therefore, we wanted to inspect their roles in immune cell infiltration. We performed ssGSEA analyses to calculate the abundance of different immune cells (Fig. 3e). Surprisingly, MMRC B and C, but especially MMRC B, were significantly enriched in immune cell infiltration containing natural killer cells, activated $\mathrm{CD} 4^{+} \mathrm{T}$ cells, dendritic cells, activated $\mathrm{CD} 8^{+} \mathrm{T}$ cells, macrophages and activated $B$ cells et al. However, MMRC $B$ and $C$ failed to present the matching survival advantages (Fig. 3b). Chen DS and I Mellman had reported that tumors also could reflect 
immunosuppressive status, even though they were accompanied by abundant immune cells. These immune cells were blocked outside of tumors by the surrounding stromal cells instead of traversing the parenchyma. It was the activation of stroma that prevented T cells from exerting their beneficial functions [33]. We offered our hypothesis that the same might apply to MMRC B. Subsequently, we calculated the stromal score and immune score using R package "Estimate". As shown in Fig. 3f, the stromal and immune score of MMRC B was remarkably higher than other clusters. What's more, from the results of GSVA, some stromal activation pathways such as EMT signaling pathway, angiogenesis, ECM receptor interaction and cytokine receptor interaction were remarkably enriched in MMRC B (Fig. 3c, Additional 1: Figure S4A). These results provided further support for our hypothesis. Next, we further analyzed the correlations between methylation modification regulators and immune cells (Additional 1: Figure S5A-D). Our previous results indicated that IGF2BP3 was more meaningful for prognosis and might act as important nodes to negatively regulate the network (Fig. 2c). We further observed that IGF2BP3 was positively related to numerous immune cells, such as activated $\mathrm{CD} 4^{+} \mathrm{T}$ cells, activated dendritic cells, CD56 Bright NK cells and macrophages (Additional 1: Figure S5B). Moreover, the "Estimate" package was used to quantify the difference of immune cell infiltration abundance between high and low expression of IGF2BP3. As shown in Fig. 3a and 3g, the expression of IGF2BP3 was much higher in MMRC B and the upregulated expression of IGF2BP3 presented a higher immune score. This implied that ccRCC patients with high expression of IGF2BP3 such as MMRC B presented a significantly higher level of immune cell infiltration. Meanwhile, all the other regulators presented negative correlations but only IGF2BP3 showed significantly opposite correlations with CD56 ${ }^{\text {Bright }}$ NK cells $(P<0.001)$. Recent studies paid more attention to the immunoregulatory functions of CD $56^{\text {Bright }} \mathrm{NK}$ cells and the mechanism of different methylation modifications regulating the distribution and function of CD56 ${ }^{\text {Bright }}$ NK cells [34-36]. The enrichment of CD56 ${ }^{\text {Bright }}$ NK cells depended on the expression of chemokine receptors, such as CCR7, CD62L and CXCR3 [37]. At the same time, CD56 ${ }^{\text {Bright }}$ NK cells and dendritic cells (DCs) can form a positive feedback regulation to enhance their immune function by promoting IL12, IL15, IL18 and IFN-y expression [38]. In addition, IL-2 was secreted from T cells to regulate the function of CD56 ${ }^{\text {Bright }} \mathrm{NK}$ cells. In turn, CD56 ${ }^{\text {Bright }}$ NK cells can inhibit the activation of T cells [39]. We analyzed the expression of these inducing factors (Fig. 3h). Our results suggested that the high expression of IGF2BP3 resulted in the comprehensively increasing expression of chemokine receptors and enhanced the abundance of CD56 Bright NK cells, consistent with previous analyses. The upregulation of other interacting factors in high IGF2BP3 group indicated that the high expression of IGF2BP3 might regulate immune activity and promote immune-cells interactions. Meanwhile, high expression of IGF2BP3 was associated closely with

PD-L1 and might affect the response to immunotherapy. From these, we speculated the IGF2BP3 mediated methylation modification could strengthen anti-tumor immune responses by activating immune cells and immune-related pathways.

\section{Construction of methylation modification regulator signature}

To further explore the potential functional and biological characteristics of the different clusters, we performed differential expression analyses between different clusters respectively and drew Venn 
diagrams to identify 2856 differential expression genes (Fig. 4a). Subsequently, we performed GO enrichment analyses using the differential expression genes. Top 10 significant enrichments were presented in Fig. 4b. We found that these genes related to pathways like antigen processing and presentation, type I interferon production, RNA splicing and epigenetic modification. This further confirmed that methylation modification regulators played an important role in immune infiltration of the tumor microenvironment. We also performed univariate Cox regression analyses among the 2856 genes, and there were 2170 genes that had prognostic value. To further verify the accuracy of our classification method, we used the R package "ConsensusClusterPlus" to perform unsupervised clustering based on the expression of 2170 DEGs. Consistent with regulator clusters, this analysis also divided $1033 \mathrm{ccRCC}$ patients into 4 subtypes, which were named as methylation-modification-gene-subtype A-D (MMGS A-D). Related to MMRC C, nearly all regulators were remarkably downregulated in MMGS C. The expression of most regulators was elevated in MMGS D, except for a few upregulated genes in MMGS A (Fig. 4C). Interestingly, in line with regulator clusters, gene subtypes $B$ and $C$ also presented high abundances of immune infiltration but demonstrated much poorer prognosis (Fig. 4d, Additional 1: Figure S4G). The concordance of function and prognosis in different clusters revealed the effectiveness and accuracy of our classification method. Due to the heterogeneity of different modifications and different individuals, these clusters based on a large patient population failed to accurately predict the specific situation of individual patients. To calculate the quantitative indicators of the landscape of methylation modification regulators, we used machine learning algorithm to perform PCA analyses. Based on the expression of 2170 DEGs, we obtained a score for each ccRCC patient which was named as methylation modification regulators score (MMR Score). The alluvial diagram presented the transformation of each individual patients' attribute (Fig. 4e). Its prognostic value was identified by Kaplan-Meier analysis (log-rank, $\mathrm{P}<0.001)$. As shown in Fig. 4f, ccRCC patients with high MMR Score had a poor prognosis. In line with this, the increasing MMR Score was accompanied by worse clinical outcomes, including higher degrees of tumor grade and tumor stage, especially pathological T stage (Fig. 4g-4i).

\section{Characteristics of MMR Score in immune checkpoints and somatic mutation}

Immune checkpoint blockade treatment provided a huge breakthrough in cancer therapy. Before the initiation of immunotherapy, the results of genetic testing were necessary to guide clinical medication better, including the expression of checkpoints, tumor mutation burden (TMB) and microsatellite instability (MSI). We firstly evaluated the condition of immune activation in cCRCC patients. CD8A, TNF, TBX2, PRF1, GZMA, GZMB, CXCL9 and CXCL10 were selected as immune activity relevant signatures [40]. As shown in Fig. 5a-5b, most of these immune activity related genes were significantly downregulated in high MMR Score group and exhibited strong negative correlations with MMR Score. It might indicate a condition of immunosuppression and reasons for poor prognosis in high MMR Score group. Meanwhile, we also evaluated the expression of common immune checkpoint blockades such as PD-1 and CTLA4 [41]. We found that PD-1 was remarkably upregulated in high MMR Score group (Fig. 5a$5 b)$. Even though the efficacy of immunotherapy did not directly relate to the expression of checkpoints, but it still indirectly implied that ccRCC patients with high MMR Score might be candidates for anti-PD-1 immunotherapy. Survival analyses showed that high expression of PD-1 presented a worse prognosis 
(Fig. 5c). Combined with MMR Score, the low-PD-1-low-MMRScore group showed a significant survival advantage and provided a more excellent predictive value (Fig. $5 \mathrm{~d}$ ). More and more evidence has shown that the high frequencies of TMB and MSI could increase immune cell infiltration and induce them to specifically recognize and eliminate cancer cells $[42,43]$. In the clinical trial (KEYNOTE 012), high frequencies of TMB were confirmed to be able to increase the response to PD-L1 blockades and improve patient outcomes [44]. As shown in Fig. 5e and 5g, we found that ccRCC patients in high TMB status were accompanied by high MMR Score and poor prognosis. The frequencies of TMB have shown a positive correlation $(\mathrm{R}=0.18, \mathrm{P}<0.05)$ with MMR Score (Fig. 5f). And the combination of high TMB and high MMR Score showed the worst survival outcome (Fig. $5 \mathrm{~h}$ ). The results provided more evidence to imply that ccRCC patients with high MMR Score might be more suitable for immunotherapy. The condition of MSI also drew our attention. Unlike what we expected, the alteration was very slight and failed to reach statistical significance in the low and high MMR Score groups (Additional 1: Figure S5E-F). There was also no statistical significance for survival analysis (Additional 1: Figure S5G). In general, most results indirectly suggested that MMR Score might act as a potential predictive biomarker and patients with a high MMR Score were more likely to derive benefits from immunotherapy, especially anti-PD-1 therapy. To further explore the distribution of somatic mutation, the "Maftools" package was used to present the distribution differences of altered genes. As shown in Fig. 5i-5j, the high MMR Score group had a higher overall mutational frequency. The alteration frequencies of VHL (45\%), TTN (17\%), SETD2 (15\%) and BAP1 (10\%) were remarkably higher than in the low MMR Score group. These alterations provided new perspective on molecular mechanisms and choices for treatment. It also helped us better understand the mechanism of somatic mutation in different dimensional methylation modifications and the potential predictive value of the MMR Score in immunotherapy.

\section{The role of MMR Score in response to immunotherapy}

ICB therapies represented by anti-PD-L1 and anti-PD-1, which play a key role in blocking the suppression of $T$ cells, has shown better outcomes in cancer treatment, but the efficacy is not available for each individual. The specific criteria of selection and application were still unknown, but necessary. To further verify the accuracy and predictive value of methylation modification model in response to immunotherapy, two immunotherapy cohorts were included in analyses. In the ccRCC immunotherapy cohort (PMID: 32472114), 172 patients who had received Nivolumab (anti-PD-1) treatment were assigned low and high MMR Score and their objective responses were divided into CR/PR (CR: complete response; PR: partial response) and SD/PD (SD: stable disease; PD: progressive disease) groups [45]. It was a pleasant surprise that patients with high MMR Score showed higher rates of therapeutic advantage and clinical benefits (Fig. 6a). At the same time, patients who developed metastases exhibited higher MMR Score (Fig. 6b). We also further validated our analyses in the IMvigor210 immunotherapy cohort (urothelial cancer patients received anti-PD-L1treatment). The results suggested that patients with high MMR Score exhibited similar clinical benefits (Fig. 6c-6d). Especially in distant metastatic lesions, such as renal pelvis and ureter, MMR Score presented more excellent value to guide immunotherapy (Fig. 6e$6 \mathrm{~g}$ ). In consideration of the relationship between MMR Score and tumor microenvironment, we used the "Cibersort" package to quantify the abundance of immune cells. Compared with SD/PD group, dendritic 
cells, mast cells and macrophages presented significant differences in immune infiltration, and macrophages decreased in CR/PR group (Fig. 6h). At the same time, rather than in low MMR Score patients, the abundance of macrophages downregulated in high MMR Score patients (Fig. 6i). And the M1 and M2 marker genes were also remarkable downregulated in high MMR Score group (Fig. 6j). This gave us new inspiration. Recent studies have shown that the PD-1 monoclonal antibodies could be captured by macrophages and failed to exert its anticancer effects [46]. It implied that the dysregulation of macrophages might play a crucial role in the tolerance in immune checkpoint blocking therapies. To further investigate the molecular mechanism between macrophages and anti-PD-1/PD-L1 immunotherapy would provide a new approach to improve the efficacy of immunotherapy.

\section{Discussion}

Transcription regulation is an important and complex biological process. It was generally known that methylation modification, the most common component of epigenetics, could give rise to stable and heritable phenotypes without changes of nucleotide sequences [5]. Accumulating evidence showed that dysregulation of methylation modifications mediated by corresponding regulators played an essential role in tumorigenesis, tumor microenvironment disorder, aberrant immune response and drug resistance [6]. However, a great deal of studies only paid attention to single regulator's biological function and the changes of single TME infiltration cell type. The cross-talk of different dimensional methylation modifications and comprehensive characterizations of TME cell infiltration mediated by interactions of multiple regulators were still not fully explored and understood. In our study, we aggregated and analyzed the 9 most common modifications from different dimension and revealed transcriptional and genetic alterations of 104 modification regulators in CcRCC patients. We also constructed a prognosis-correlation network to further explore their interactions between different modifications (Fig. 2C). PRMT8, IGF2BP2 and especially IGF2BP3 were identified as key negative nodes in the network. Meanwhile, the high expression of IGF2BP3 could enhance the abundance of CD56 Bright $N K$ cells by inducing the upregulation of chemokine receptors. IGF2BP3 could also regulate immune activity and promote immune-cells interactions by increasing the expression of interacting factors.

Subsequently, on the basis of these regulators, we identified four distinct methylation modification regulator clusters. These clusters presented significantly distinct clinical outcomes and characterizations of TME cell infiltration. We found that innate immunity was markedly activated in MMRC C, while MMRC $B$ exhibited significant adaptive immunity activation. But both MMRC B and $C$ failed to present corresponding survival advantage. In melanoma and other tumor types, tumor tissues were infiltrated with substantial amounts of $\mathrm{CD}^{+} \mathrm{T}$ cells, which formed immune-inflamed or hot-tumor phenotype and responded better to immune checkpoint blockade therapy [40, 47, 48]. But in advanced ccRCC, the situation is just opposite. We further found that the stromal scores of MMRC B and $C$ were significantly higher. The results of GSVA also revealed that MMRC B and C were markedly enriched in stromal activation and tumor proliferation, metastasis pathway including EMT signaling pathway, angiogenesis, inflammatory response, KRAS and P53 signaling pathway. This implied that the response to 
immunotherapy might be not completely predicted by the immune phenotypes. An excess of stromal cell infiltration can interfere with intercellular communication between immune cells and tumor cells and disturb the immune activity and immune response [33].

Additionally, we screened DEGs between different clusters. Enrichment analyses showed that these genes were associated with methylation modifications, immune response-related pathways, and were seen as methylation modification related signature genes. Based on prognosis-related DEGs, we have identified 4 methylation modification gene subtypes. Similar to regulator clusters, these methylation modification related gene subtypes were also related to stromal activation and immune infiltration. Once again, it confirmed that methylation modification patterns played a significant role in the formation of different TME landscapes. Consequently, integrative analysis of the cross-talk of different dimensional methylation modifications mediated by interactions of multiple regulators was greatly warranted.

In consideration of the heterogeneity of different patients, individual quantification of methylation modification patterns for each patient is urgently needed. The score model based on individual heterogeneity to predict individual outcome has been well used in breast cancer and colorectal cancer [49, 50]. For this, based on the modification related genes, we established a scoring model which was named MMR Score to assess the methylation modification characterizations of each patient. Patients with high MMR Score were more inclined to experience malignant progression and worse overall survival.

Immune checkpoint blockade treatment provided a huge breakthrough in cancer therapy. A notable limitation of immune checkpoint blockade treatment is the limited applicable populations. Before the initiation of immunotherapy, the results of genetic testing were necessary to better guide clinical medication, including the expression of checkpoints, TMB and MSI [42-44]. Our results showed that, between high and low MMR Score groups, there were many significant mutational frequency differences. The alteration frequencies of multiple genes were remarkably higher in the high MMR Score group. Furthermore, we also found that TMB was significantly increased in the high MMR Score group, which indicated that patients with high MMR Score might be more sensitive to immunotherapy. The combination of TMB and MMR Score could better predict overall survival and guide clinical treatment. But MSI lacked correlation with MMR Score, and there was no difference in survival between high and low MSI groups. This implied that MSI had no predictive value in ccRCC. Through comprehensive comparison of common immune checkpoint blockade expression, gene mutation status and prognostic outcome, MMR Score was considered as a meaningful biomarker which could effectively predict the response to immune checkpoint blockade treatment. In two immunotherapy multicenter cohorts, we separately verified the predictive value of MMR Score in CCRCC patients and found that MMR Score was remarkably increased in responders. The predictive value of MMR Score was also not merely limited to ccRCC patients. In other common urologic malignancies such as urothelial cancer, the validation of results both achieved excellent agreement. The abundance of immune infiltration between responders and nonresponders was compared with high and low MMR Score groups. Macrophages were one of the few immune cell types that showed a significant difference. Recent studies have shown that the PD-1 monoclonal antibodies could be captured by macrophages while failing to exert their anticancer effects 
[46]. The dysregulation of macrophages might play an important role in the tolerance of ICB therapies, but it still requires further investigation. Besides, results of the current study still need to be validated in a larger immunotherapy cohort. And the specific mechanisms of cross-talk of different dimensional methylation modifications require more experiments to explore further.

In general, we comprehensively explored the landscape of different dimensional methylation modifications and constructed a clear regulated network. At the same time, the characterizations of methylation modification patterns were quantified as individual MMR Score. MMR Score can be used to evaluate the tumor pathology, malignancy grade and states of TME infiltration. Furthermore, it can help to identify the applicable patients for immunotherapy and better guide clinical personalized cancer treatment.

\section{Conclusions}

In conclusion, we comprehensively and systematically analyzed the extensive regulatory mechanisms of cross-talk of different dimensional methylation modifications and revealed their effects on TME and cCRCC prognosis. The differences of methylation modifications were non-negligible factors which lead to the complexity and heterogeneity of individual TME. The integrated quantification of individual methylation modification highlights the important clinical implications of cross-talk of different dimensional methylation modifications and can better guide clinical personalized cancer treatment.

\section{Abbreviations}

carRNA: chromosome-associated regulatory RNA; ccRCC: clean cell renal cell carcinoma; CNV: copy number variation; DEG: differentially expressed gene; GEO: Gene Expression Omnibus; ICB: immune checkpoint blockade; KIRC: kidney renal clear cell carcinoma; MMGS: methylation modification gene subtype; MMRC: methylation modification regulator cluster; MMR Score: methylation modification regulator score; MSI: microsatellite instability; TCGA: The Cancer Genome Atlas; TMB: tumor mutation burden; TME: tumor microenvironment; TPM: transcripts per kilobase million.

\section{Declarations}

\section{Acknowledgements}

We thank Dr. Michael Strickland (University of California, Los Angeles) for checking the grammar of this article.

\section{Authors' contributions}

Xin Qin and Ling Pan analyzed the data and selected the analyzed results. Yidong Fan, Zhiqing Fang and Lei Yan designed the project and wrote the paper. All authors read and approved the final manuscript. 


\section{Funding}

This work was supported by the Chinese Postdoctoral Science Foundation (2019M650164), Shandong Provincial Natural Science Foundation (No. ZR2020QH245).

\section{Availability of data and materials}

All the clinical information and data matrix can be downloaded from GEO database

(https://www.ncbi.nlm.nih.gov/geo/), Array Express database (https://www.ebi.ac.uk/arrayexpress/) and TCGA database (https://portal.gdc.cancer.gov/). Immunotherapy dataset: IMvigor210 cohort, an anti-PDL1cohort, and corresponding clinical information came from http://researchpub.gene.com/IMvigor210Corebiologies; Another immunotherapy cohort dataset was downloaded from a published article (PMID: 32472114) including the datasets of ccRCC patients received anti-PD1 treatment [24].

\section{Ethics approval and consent to participate}

The clinical information and data matrix came from public database which obtained patients' consent.

\section{Consent for publication}

Not applicable.

\section{Competing interests}

The authors have declared that no competing interest exists.

\section{References}

1. Siegel R, Miller K, Jemal A. Cancer statistics, 2020. CA Cancer J Clin. 2020;70:7-30.

2. Scelo G, Larose T. Epidemiology and Risk Factors for Kidney Cancer. J Clin Oncol. 2018;36:JCO2018791905.

3. Capitanio U, Montorsi F. Renal cancer. Lancet. 2016;387:894-906.

4. Barata P, Rini B. Treatment of renal cell carcinoma: Current status and future directions. CA Cancer J Clin. 2017;67:507-24.

5. Kietrys A, Kool E. Epigenetics. A new methyl mark on messengers. Nature. 2016;530:423-4.

6. Chen Y, Hong T, Wang S, Mo J, Tian T, et al. Epigenetic modification of nucleic acids: from basic studies to medical applications. Chem Soc Rev. 2017;46:2844-72.

7. Klemm S, Shipony Z, Greenleaf W. Chromatin accessibility and the regulatory epigenome. Nat Rev Genet. 2019;20:207-20.

8. Greenberg M, Bourc'his D. The diverse roles of DNA methylation in mammalian development and disease. Nat Rev Mol Cell Biol. 2019;20:590-607. 
9. Robertson K. DNA methylation and chromatin - unraveling the tangled web. Oncogene. 2002;21:5361-79.

10. Roundtree I, Evans M, Pan T, He C. Dynamic RNA Modifications in Gene Expression Regulation. Cell. 2017;169:1187-200.

11. Liu J, Dou X, Chen C, Chen C, Liu C, et al. $\mathrm{N}^{6}$-methyladenosine of chromosome-associated regulatory RNA regulates chromatin state and transcription. 367. New York: Science; 2020. pp. 580-6.

12. Bannister A, Kouzarides T. Regulation of chromatin by histone modifications. Cell Res. 2011;21:38195.

13. Michalak E, Burr M, Bannister A, Dawson M. The roles of DNA, RNA and histone methylation in ageing and cancer. Nat Rev Mol Cell Biol. 2019;20:573-89.

14. Huang $\mathrm{H}$, Weng $\mathrm{H}$, Zhou $\mathrm{K}$, Wu T, Zhao $\mathrm{B}$, et al. Histone $\mathrm{H} 3$ trimethylation at lysine 36 guides $\mathrm{m}^{6} \mathrm{~A}$ RNA modification co-transcriptionally. Nature. 2019;567:414-9.

15. Li Y, Xia L, Tan K, Ye X, Zuo Z, et al. $\mathrm{N}^{6}$-Methyladenosine co-transcriptionally directs the demethylation of histone H3K9me2. Nat Genet. 2020;52:870-7.

16. Foster B, Stolz P, Mulholland C, Montoya A, Kramer H, et al. Critical Role of the UBL Domain in Stimulating the E3 Ubiquitin Ligase Activity of UHRF1 toward Chromatin. Mol Cell. 2018;72:739 52.e9..

17. Curran M, Montalvo W, Yagita H, Allison J. PD-1 and CTLA-4 combination blockade expands infiltrating $T$ cells and reduces regulatory $T$ and myeloid cells within B16 melanoma tumors. Proc Natl Acad Sci U S A. 2010;107:4275-80.

18. Cabrera R, Szabo G. Another armed CD4(+) T cell ready to battle hepatocellular carcinoma. Hepatology. 2013;58:1-3.

19. Jiang Y, Zhang Q, Hu Y, Li T, Yu J, et al. ImmunoScore Signature: A Prognostic and Predictive Tool in Gastric Cancer. Ann Surg. 2018;267:504-13.

20. Han D, Liu J, Chen C, Dong L, Liu Y, et al. Anti-tumour immunity controlled through mRNA $\mathrm{m}^{6} \mathrm{~A}$ methylation and YTHDF1 in dendritic cells. Nature. 2019;566:270-4.

21. Wang $H$, Hu X, Huang M, Liu J, Gu Y, et al. Mettl3-mediated mRNA m ${ }^{6}$ A methylation promotes dendritic cell activation. Nat Commun. 2019;10:1898.

22. Zhang X, Shi M, Chen T, Zhang B. Characterization of the Immune Cell Infiltration Landscape in Head and Neck Squamous Cell Carcinoma to Aid Immunotherapy. Mol Ther Nucleic Acids. 2020;22:298309.

23. Johnson W, Li C, Rabinovic A. Adjusting batch effects in microarray expression data using empirical Bayes methods. Biostatistics. 2007;8:118-27.

24. Mariathasan S, Turley S, Nickles D, Castiglioni A, Yuen K, et al. TGF $\beta$ attenuates tumour response to PD-L1 blockade by contributing to exclusion of T cells. Nature. 2018;554:544-8.

25. Wilkerson M, Hayes D. ConsensusClusterPlus: a class discovery tool with confidence assessments and item tracking. Bioinformatics. 2010;26:1572-3. 
26. Hänzelmann S, Castelo R, Guinney J. GSVA: gene set variation analysis for microarray and RNA-seq data. BMC Bioinformatics. 2013;14:7.

27. Yoshihara K, Shahmoradgoli M, Martínez E, Vegesna R, Kim H, et al. Inferring tumour purity and stromal and immune cell admixture from expression data. Nat Commun. 2013;4:2612.

28. Newman A, Liu C, Green M, Gentles A, Feng W, et al. Robust enumeration of cell subsets from tissue expression profiles. Nat Methods. 2015;12:453-7.

29. Ritchie M, Phipson B, Wu D, Hu Y, Law C, et al. limma powers differential expression analyses for RNA-sequencing and microarray studies. Nucleic Acids Res. 2015;43:e47.

30. Sotiriou C, Wirapati P, Loi S, Harris A, Fox S, et al. Gene expression profiling in breast cancer: understanding the molecular basis of histologic grade to improve prognosis. J Natl Cancer Inst. 2006;98:262-72.

31. Zaccara S, Ries R, Jaffrey S. Reading, writing and erasing mRNA methylation. Nat Rev Mol Cell Biol. 2019;20:608-24.

32. Zhao Y, Garcia B. Comprehensive Catalog of Currently Documented Histone Modifications. Cold Spring Harb Perspect Biol. 2015;7:a025064.

33. Chen D, Mellman I. Elements of cancer immunity and the cancer-immune set point. Nature. 2017;541:321-30.

34. Poli $A$, Michel $T$, Thérésine $M$, Andrès $E$, Hentges $F$, et al. CD56bright natural killer (NK) cells: an important NK cell subset. Immunology. 2009;126:458-65.

35. Freud A, Mundy-Bosse B, Yu J, Caligiuri M. The Broad Spectrum of Human Natural Killer Cell Diversity. Immunity. 2017;47:820-33.

36. Michel T, Poli A, Cuapio A, Briquemont B, Iserentant G, et al. Human CD56bright NK Cells: An Update. J Immunol. 2016;196:2923-31.

37. Dubois S, Conlon K, Müller J, Hsu-Albert J, Beltran N, et al. IL15 Infusion of Cancer Patients Expands the Subpopulation of Cytotoxic CD 56 NK Cells and Increases NK-Cell Cytokine Release Capabilities. Cancer Immunol Res. 2017;5:929-38.

38. Carrega P, Morandi B, Costa R, Frumento G, Forte G, et al. Natural killer cells infiltrating human nonsmall-cell lung cancer are enriched in CD56 bright CD16(-) cells and display an impaired capability to kill tumor cells. Cancer. 2008;112:863-75.

39. Gogali F, Paterakis G, Rassidakis G, Liakou C, Liapi C. CD3(-)CD16(-)CD56(bright) immunoregulatory NK cells are increased in the tumor microenvironment and inversely correlate with advanced stages in patients with papillary thyroid cancer. Thyroid. 2013;23:1561-8.

40. Hugo W, Zaretsky J, Sun L, Song C, Moreno B, et al. Genomic and Transcriptomic Features of Response to Anti-PD-1 Therapy in Metastatic Melanoma. Cell. 2016;165:35-44.

41. Ayers M, Lunceford J, Nebozhyn M, Murphy E, Loboda A, et al. IFN-y-related mRNA profile predicts clinical response to PD-1 blockade. J Clin Invest. 2017;127:2930-40. 
42. Samstein R, Lee $C$, Shoushtari A, Hellmann M, Shen R, et al. Tumor mutational load predicts survival after immunotherapy across multiple cancer types. Nat Genet. 2019;51:202-6.

43. Rodriquenz M, Roviello G, D'Angelo A, Lavacchi D, Roviello F, et al. MSI and EBV Positive Gastric Cancer's Subgroups and Their Link With Novel Immunotherapy. J Clin Med. 2020;9:1427.

44. Nanda R, Chow L, Dees E, Berger R, Gupta S, et al. Pembrolizumab in Patients With Advanced TripleNegative Breast Cancer: Phase Ib KEYNOTE-012 Study. J Clin Oncol. 2016;34:2460-7.

45. Braun D, Hou Y, Bakouny Z, Ficial M, Sant' Angelo M, et al. Interplay of somatic alterations and immune infiltration modulates response to PD-1 blockade in advanced clear cell renal cell carcinoma. Nat Med. 2020;26:909-18.

46. Arlauckas S, Garris C, Kohler R, Kitaoka M, Cuccarese M, et al. In vivo imaging reveals a tumorassociated macrophage-mediated resistance pathway in anti-PD-1 therapy. Sci Transl Med. 2017;9.

47. Cho J, Hong M, Ha S, Kim Y, Cho B, et al. Genome-wide identification of differentially methylated promoters and enhancers associated with response to anti-PD-1 therapy in non-small cell lung cancer. Exp Mol Med. 2020;52:1550-63.

48. Li J, Byrne K, Yan F, Yamazoe T, Chen Z, et al. Tumor Cell-Intrinsic Factors Underlie Heterogeneity of Immune Cell Infiltration and Response to Immunotherapy. Immunity. 2018;49:178 - 93.e7..

49. Callari M, Cappelletti V, D'Aiuto F, Musella V, Lembo A, et al. Subtype-Specific Metagene-Based Prediction of Outcome after Neoadjuvant and Adjuvant Treatment in Breast Cancer. Clin Cancer Red. 2016;22:337-45.

50. Bramsen J, Rasmussen M, Ongen H, Mattesen T, Ørntoft M, et al. Molecular-Subtype-Specific Biomarkers Improve Prediction of Prognosis in Colorectal Cancer. Cell Rep. 2017;19:1268-80.

\section{Figures}



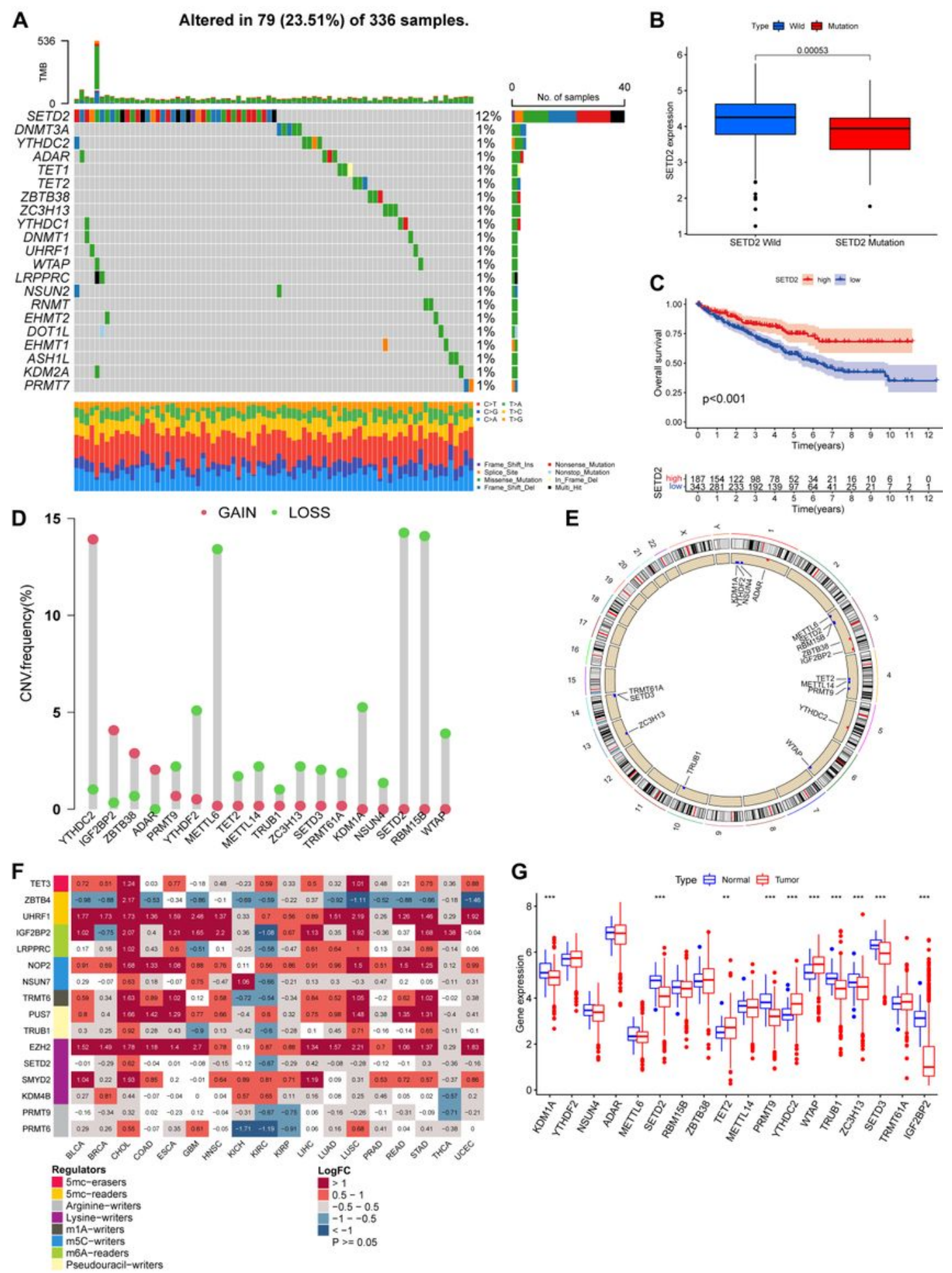

\section{Figure 1}

Landscape of genetic and transcriptional changes of methylation modification regulators in ccRCC. (A) The mutation frequency of methylation modification regulators in cCRCC patients. TMB was presented in the upper panel. The right panel exhibited the frequencies and types of mutation. The lower panel showed the fraction of conversions in each ccRCC patients. (B) The expression of SETD2 in wild type and mutation type. (C) Overall survival of ccRCC patients in SETD2 high (red) and low (blue) expression group 
( $P<0.05$, Log-rank test). (D) The CNV frequency of regulators. Green dot: CNV loss; red dot: CNV gain. (E) The distribution of CNV alteration of methylation modification regulators on chromosomes. (F) The pancancer expression of regulators which showed significant differences in TCGA-KIRC dataset. (G) The expression of regulators which showed significant CNV alteration in cCRCC patients. $*$ : $P<0.05$. **: $\mathrm{P}<0.01 . * * *: \mathrm{P}<0.001$.

A
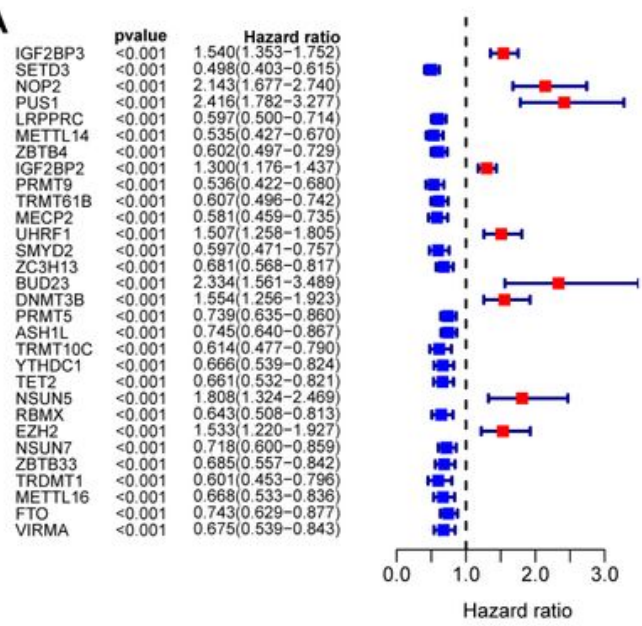

B

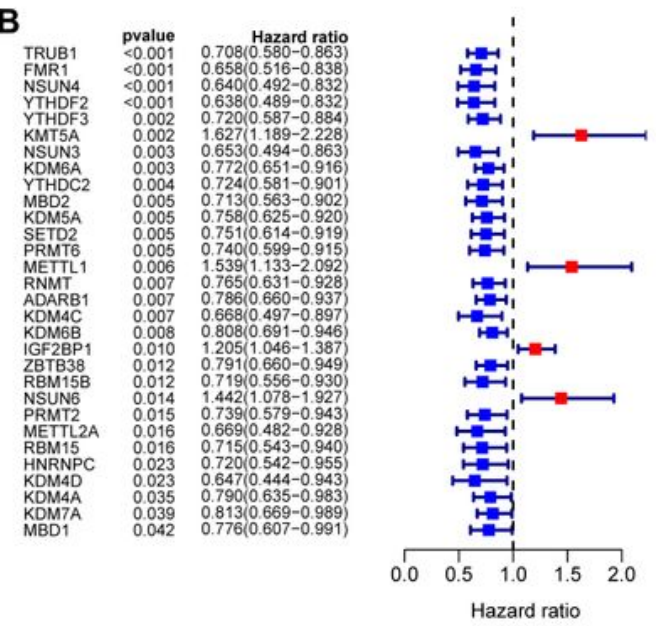

C

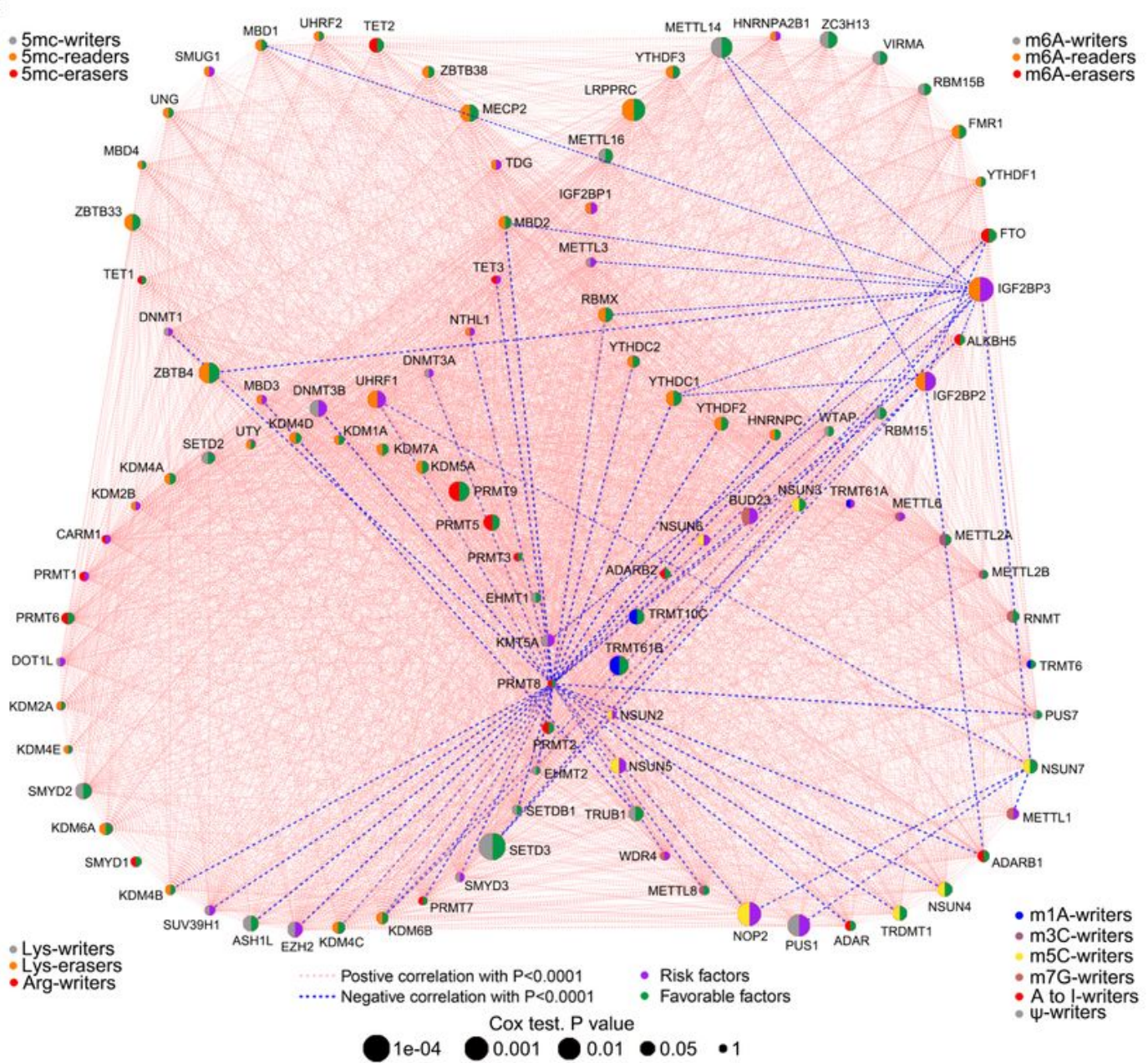

Figure 2 
Prognostic value and crosstalk patterns of different dimensional regulators. (A-B) We performed univariate Cox regression analysis to screen prognosis-related regulators in ccRCC patients $(P<0.05)$. (C) The interactions between methylation modification regulators in ccRCC patients. Size of circle showed the influence of each methylation modification regulator on prognosis. Purple half-circle (right): risk factors; green half-circle (right): favorable factors. The color of left half-circle represented their role in different modifications. The dashed presented their positive interactions (pink) and negative interactions (blue). $\mathrm{P}<0.0001$ was consider as significant co-expression relationships.
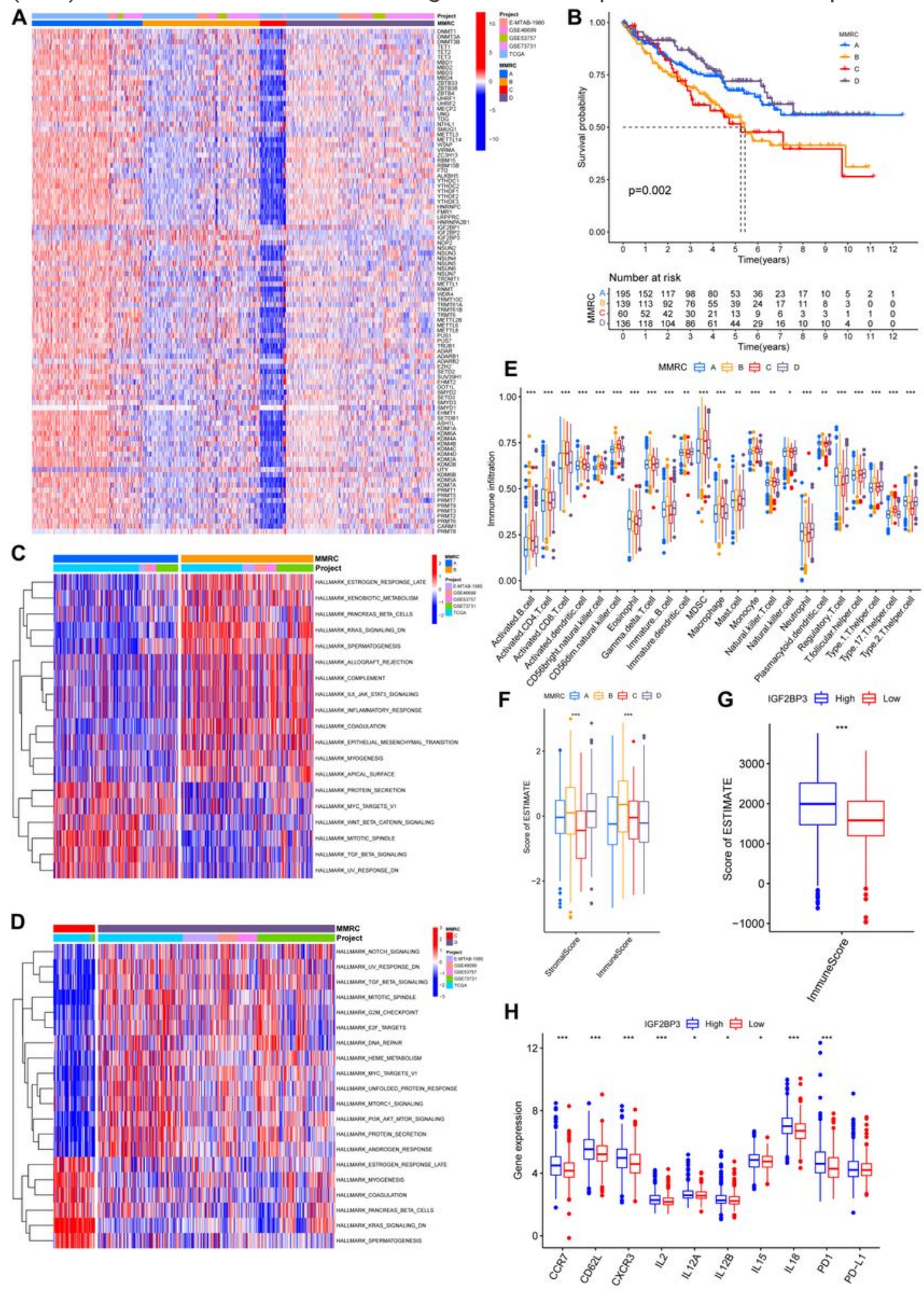


\section{Figure 3}

Distinct clusters and immune infiltration characteristics of modification regulators. (A) Unsupervised clustering analysis of 104 regulators. The project of ccRCC cohorts and MMRC type were annotated in right panel. Red: high expression. Blue: low expression. (B) Overall survival of four methylation modification regulator clusters $(P<0.05$, Log-rank test). (C-D) Heatmaps of GSVA enrichment analysis was used to present the states of different pathways in MMRC. Red: activation. Blue: inhibition. (C) MMRC A vs MMRC B; hallmark gene set. (D) MMRC $C$ vs MMRC $D$; hallmark gene set. (E) The abundance of immune cell infiltration in four MMRC patterns ( $* * *: P<0.001$; $* *$ : $P<0.01 ; *$ : $P<0.05$ ). (F) ESTIMATE score of different MMRC patterns. (G) ESTIMATE score of ccRCC patients in IGF2BP3 high and low expression group. $(\mathrm{H})$ The differential expression of chemokine receptors and other interacting factors of CD56Bright NK cells in IGF2BP3 high and low expression group. 

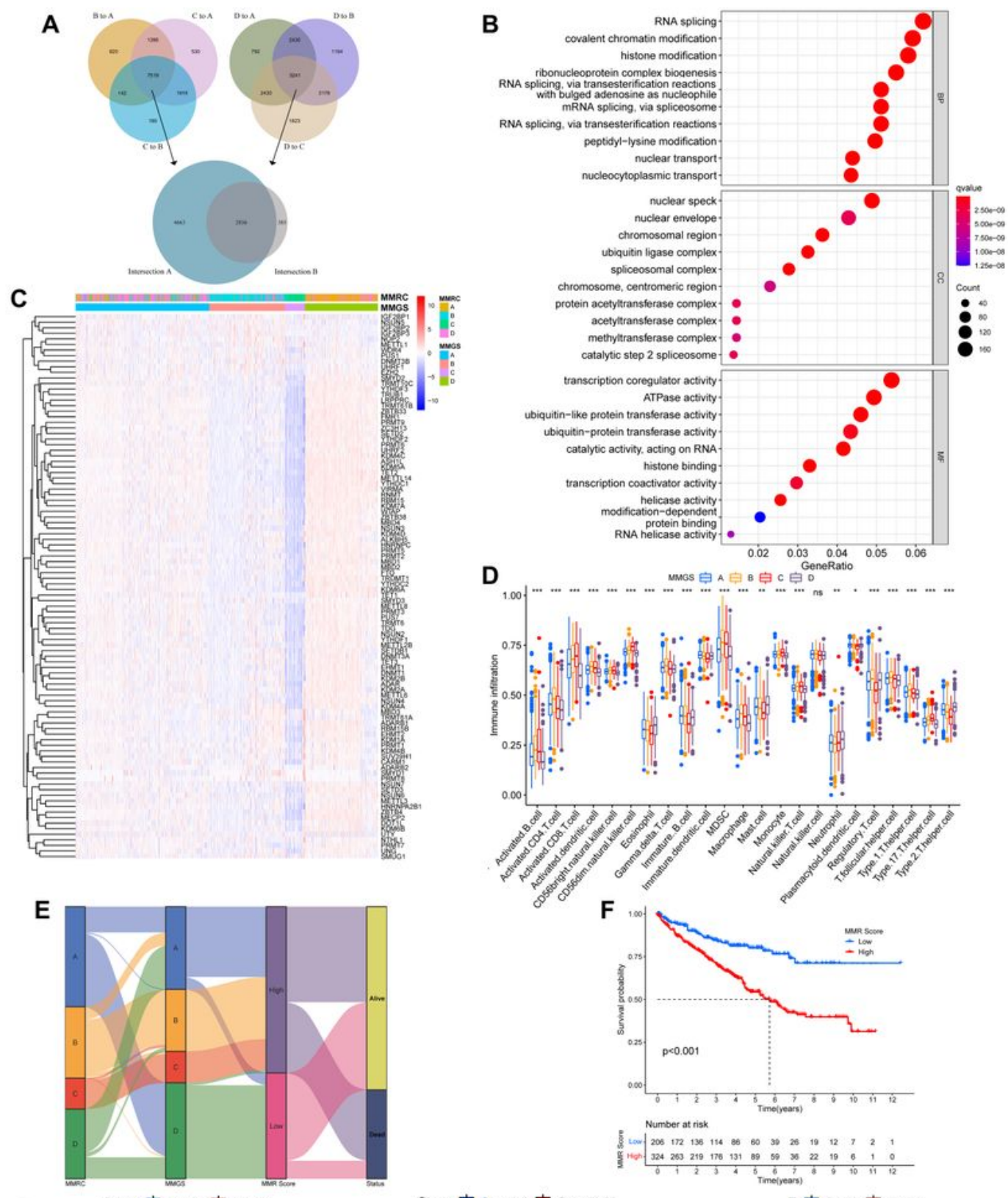

Number at tisk

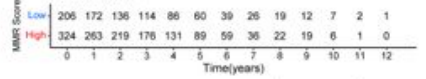
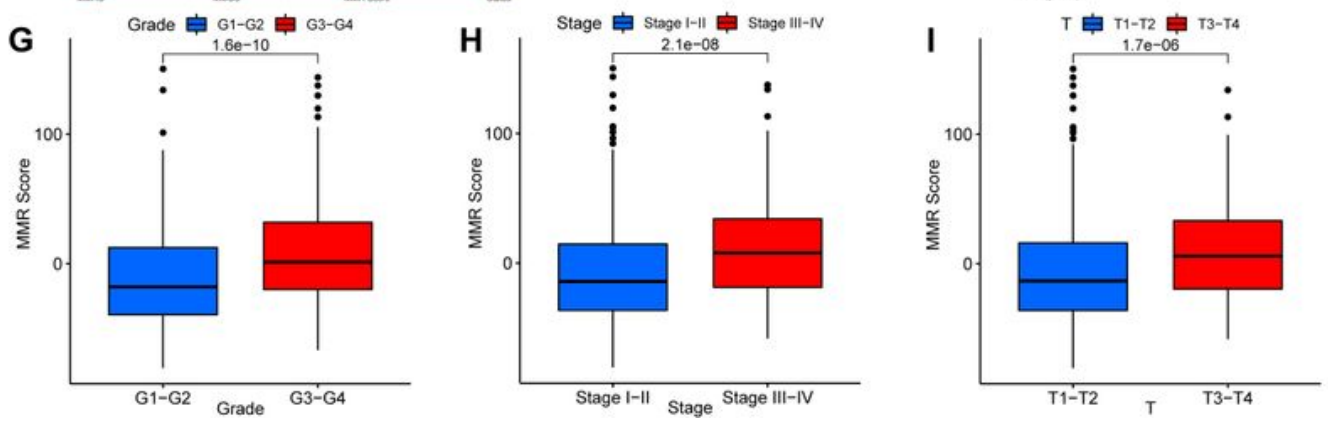

\section{Figure 4}

Construction of methylation modification regulator signature. (A) Venn diagram showed the intersection of DEGs between distinct MMRC patterns. (B) GO enrichment analyses for methylation modification related genes. The size of circle represented the count of enriched genes. (C) Unsupervised clustering analysis of methylation modification related genes. The type of MMRC and MMGS were annotated in right panel. Red: high expression. Blue: low expression. (D) The abundance of immune cell infiltration in 
four MMGS patterns. *: $P<0.05$. **: $P<0.01 . * \star *: P<0.001$. (F) Overall survival of low and high MMR Score groups using Kaplan-Meier curves $(P<0.05$, Log-rank test). (G-I) The relationships between MMR Score and clinical pathological phenotype. G: MMR Score with tumor grade; H: MMR Score with tumor stage; I: MMR Score with T stage.
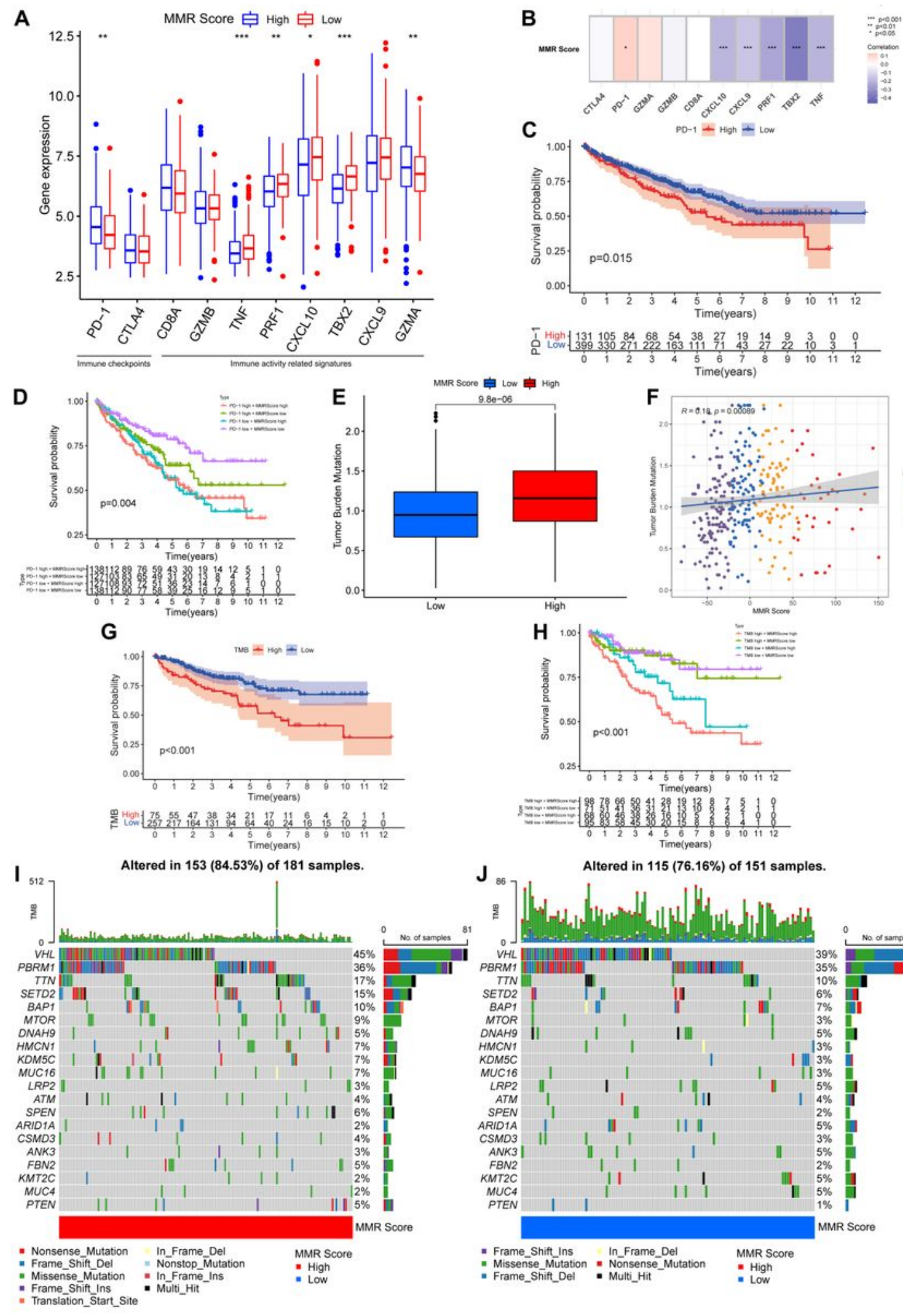

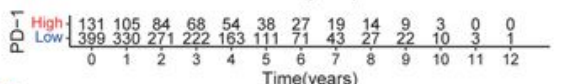

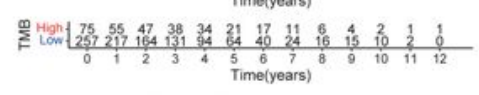

tered in $153(84.53 \%)$ of 181 samples.

\section{Figure 5}


Characteristics of MMR Score in immune checkpoints and somatic mutation. (A) The differential expression of immune activity related gene signatures and immune checkpoints in low and high MMR Score group. $*$ : $P<0.05$. **: $P<0.01$. ***: $P<0.001$. (B) The correlation analyses between immune related gene signature and MMR Score. (C) Overall survival of low and high PD-1 patient groups using KaplanMeier curves $(P<0.05$, Log-rank test). (D) Survival analysis for patients in the TCGA-KIRC cohort stratified by both MMR Score and expression of PD-1 ( $<0.05$, Log-rank test). (E) Differences in TMB between MMR Score high (red) and low (blue) group. Wilcoxon test was performed to assess the difference. $(F)$ Scatterplot was used to present the correlations between TMB and MMR Score. (G) Overall survival of low and high TMB groups using Kaplan-Meier curves $(P<0.05$, Log-rank test). $(H)$ Survival analysis for patients in the TCGA-KIRC cohort stratified by both MMR Score and TMB $(P<0.05$, Log-rank test). (I-J) Waterfall plots were constructed to present somatic mutation based on patients with high MMR Score (I) and low MMR Score (J). 

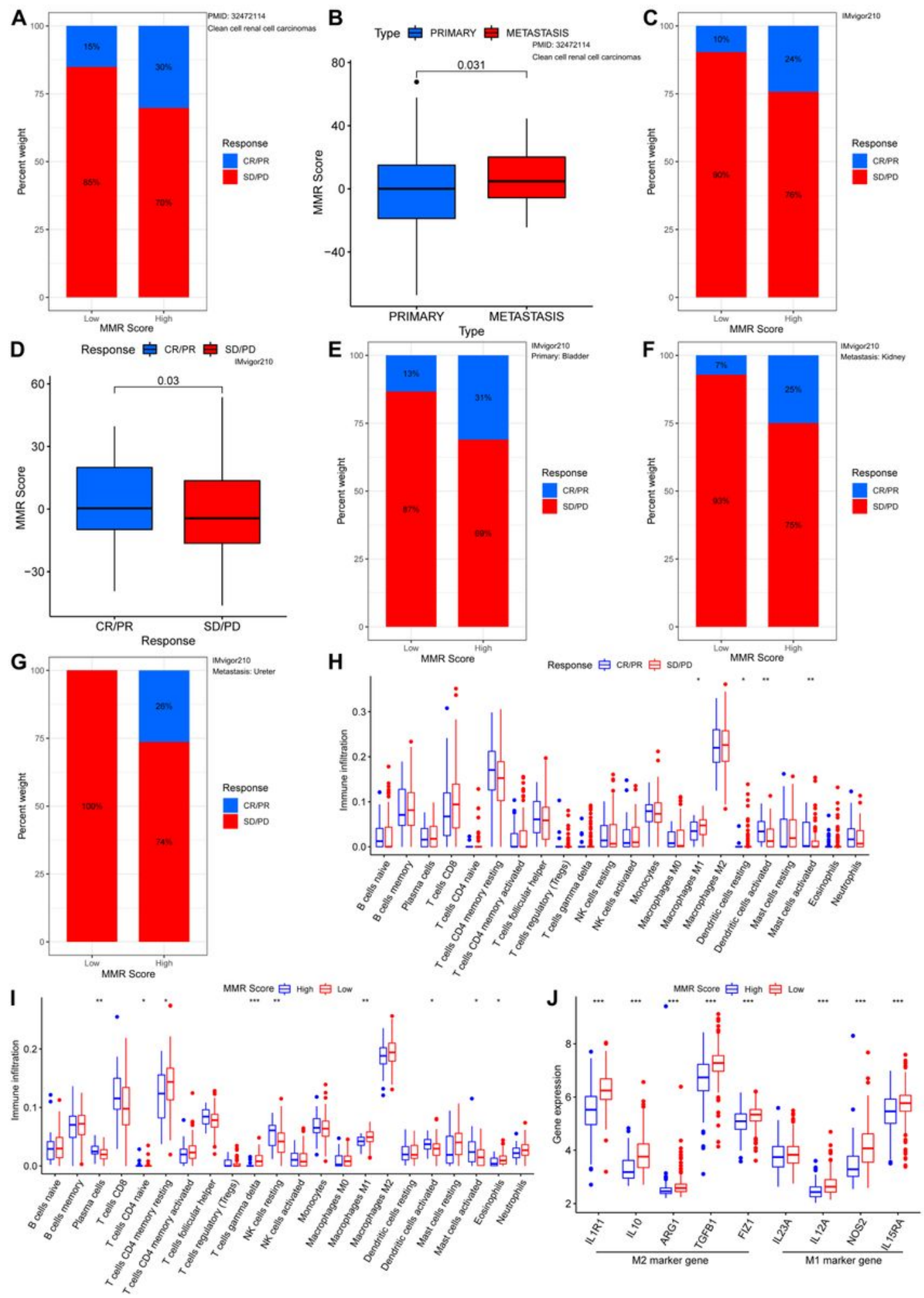

\section{Figure 6}

The role of MMR Score in response to immunotherapy. (A) Different responses to anti-PD-1 immunotherapy in high or low MMR Score groups in ccRCC immunotherapy cohort. (B) Differences in MMR Score between metastasis tumor (red) and primary tumor (blue) group. (C) Different responses to anti-PD-L1 immunotherapy in high or low MMR Score groups in IMvigor210 immunotherapy cohort. (D) Differences in MMR Score between SD/PD (red) and CR/PR (blue) group. (E-G) Different responses to 
anti-PD-L1 immunotherapy in high or low MMR Score groups. E: differences in primary bladder tissue; F: differences in metastatic kidney tissue; G: differences in metastatic ureter tissue. $(H)$ The abundance of immune cell infiltration in SD/PD and CR/PR group. ***: $P<0.001$; $* *$ : $P<0.01$; $*$ : $<0.05$. (I) The abundance of immune cell infiltration in low and high MMR Score group. $* * *$ : $<<0.001 ; * *$ : $P<0.01 ; *: P<$ 0.05. $(\mathrm{J})$ The differential expression of macrophage marker genes in high and low MMR Score group (***: $P<0.001 ; * *: P<0.01 ; *: P<0.05)$.

\section{Supplementary Files}

This is a list of supplementary files associated with this preprint. Click to download.

- Additionalfile1.pdf

- Additionalfile2.xlsx 